\title{
Trisomy 8-positive Polycythemia Vera Complicated with Intestinal Behçet's-like Disease: A New Perspective for a Clinical Approach
}

\author{
Yuki Mori ${ }^{1}$, Fumihiko Iwamoto ${ }^{1}$, Toru Kuno ${ }^{1}$, Shoji Kobayashi ${ }^{1}$, Takashi Yoshida ${ }^{1}$, \\ Tatsuya Yamaguchi ${ }^{1}$, Shinichi Takano ${ }^{1}$, Tetsuo Kondo ${ }^{2}$, Keita Kirito ${ }^{3}$ and Nobuyuki Enomoto ${ }^{1}$
}

\begin{abstract}
:
Behçet's disease (BD) is a multisystem inflammatory disease of unknown origin. It rarely but occasionally occurs together with myelodysplastic syndrome and primary myelofibrosis. Trisomy 8 is one of the most common cytogenetic abnormalities in myeloid neoplasms; however, the association of BD with polycythemia vera (PV) and trisomy 8 has not been reported. A 70-year-old woman, diagnosed with PV and treated with hydroxyurea, had bloody stool due to multiple ulcers in the ileocecal region. Considering the lack of a response to treatment and other features, we suspected complication with intestinal Behçet's-like disease. Our case suggests relationships among $\mathrm{BD}$, trisomy 8 , and $\mathrm{PV}$.
\end{abstract}

Key words: Behcet's disease, Polycythemia vera, Trisomy 8

(Intern Med Advance Publication)

(DOI: 10.2169/internalmedicine.8395-21)

\section{Introduction}

Behçet's disease (BD) is a multisystem inflammatory disease characterized by recurrent oral ulcers, skin lesions, uveitis, and genital ulcers. Many other systems can be involved, including the gastrointestinal tract, central nervous system, cardiovascular system, and musculoskeletal system (1-3). Intestinal BD occurs in 3-60\% of patients with BD (2-4). Its clinical manifestations range widely, from mild abdominal pain to bowel perforation or massive hemorrhaging with a poor prognosis (5). Although immunosuppressive agents, such as cyclosporine, cyclophosphamide, anti-TNFalpha antibody (infliximab, adalimumab), and corticosteroids, are used to treat intestinal $\mathrm{BD}$, a standard treatment for intractable BD has not been established. Surgery is considered in patients with intestinal $\mathrm{BD}$ who are unresponsive to medical treatment or those with bowel complications, such as perforation or persistent bleeding (6).

The relationship of trisomy 8 with intestinal Behçet's-like disease and myeloid neoplasms, such as acute monocytic leukemia (7), primary myelofibrosis (PMF) (8) and myelodysplastic syndrome (MDS), has recently been reported. However, there have been no reports of cases in which intestinal Behçet's-like disease, myeloid neoplasms, and trisomy 8 all co-occurred. Most patients with symptoms of BD who also have trisomy 8 and MDS have intestinal manifestations of BD (9-11). Because trisomy 8 induces the activation of an abnormal inflammatory process and immune gene expression (12), trisomy 8 in MDS can contribute to the aggravation of intestinal ulcers in patients with BD. In fact, the intestinal lesions in BD patients with trisomy 8 are refractory to conventional medical therapy. Despite the use of various therapeutic agents, such as colchicine, 5aminosalicylates (5-ASA), corticosteroids, and immunomodulators, about $30 \%$ of BD patients with MDS involving trisomy 8 fail to respond to pharmacologic treatment (11).

Polycythemia vena (PV) is a myeloproliferative neoplasm (MPN) characterized by the excessive production of red blood cells and clonal myeloproliferation as a consequence of an acquired mutation of the Janus kinase 2 (JAK2) gene (13). Although PV, PMF, and MDS are a group of

${ }^{1}$ First Department of Internal Medicine, Faculty of Medicine, University of Yamanashi, Japan, ${ }^{2}$ Department of Pathology, Faculty of Medicine, University of Yamanashi, Japan and ${ }^{3}$ Department of Hematology and Oncology, University of Yamanashi, Japan Received: July 23, 2021; Accepted: September 15, 2021; Advance Publication by J-STAGE: November 6, 2021 Correspondence to Dr. Tatsuya Yamaguchi, ytatsuya@yamanashi.ac.jp 
Table 1. Patient Laboratory Data at PV Diagnosis.

\begin{tabular}{|c|c|c|}
\hline Test name & Value & Units \\
\hline \multicolumn{3}{|l|}{ Biochemistry } \\
\hline $\mathrm{TP}$ & \multicolumn{2}{|c|}{$8.4 \mathrm{~g} / \mathrm{dL}$} \\
\hline $\mathrm{Alb}$ & \multicolumn{2}{|c|}{$3.7 \mathrm{~g} / \mathrm{dL}$} \\
\hline T-Bil & \multicolumn{2}{|c|}{$0.5 \mathrm{mg} / \mathrm{dL}$} \\
\hline BUN & \multicolumn{2}{|c|}{$12.8 \mathrm{mg} / \mathrm{dL}$} \\
\hline $\mathrm{Cr}$ & \multicolumn{2}{|c|}{$0.66 \mathrm{mg} / \mathrm{dL}$} \\
\hline AST & \multicolumn{2}{|c|}{24 IU/L } \\
\hline ALT & \multicolumn{2}{|c|}{$20 \mathrm{IU} / \mathrm{L}$} \\
\hline LDH & \multicolumn{2}{|c|}{397 IU/L } \\
\hline$\gamma$-GTP & \multicolumn{2}{|c|}{$69 \mathrm{mg} / \mathrm{dL}$} \\
\hline CRP & \multicolumn{2}{|c|}{$0.81 \mathrm{mg} / \mathrm{dL}$} \\
\hline $\mathrm{Na}$ & \multicolumn{2}{|c|}{136 mEq/L } \\
\hline K & \multicolumn{2}{|c|}{$5.2 \mathrm{mEq} / \mathrm{L}$} \\
\hline $\mathrm{Cl}$ & \multicolumn{2}{|c|}{$102 \mathrm{mEq} / \mathrm{L}$} \\
\hline \multicolumn{3}{|l|}{ Hematology } \\
\hline WBC & \multicolumn{2}{|c|}{$20,560 / \mu \mathrm{L}$} \\
\hline $\mathrm{Hb}$ & \multicolumn{2}{|c|}{$18.0 \mathrm{~g} / \mathrm{dL}$} \\
\hline $\mathrm{RBC}$ & \multicolumn{2}{|c|}{$727 \times 10^{4} / \mu \mathrm{L}$} \\
\hline $\mathrm{Ht}$ & \multicolumn{2}{|c|}{$58.3 \%$} \\
\hline Plt & \multicolumn{2}{|c|}{$48.1 \times 10^{4} / \mu \mathrm{L}$} \\
\hline \multicolumn{3}{|l|}{ Other } \\
\hline EPO & \multicolumn{2}{|c|}{$8.4 \mathrm{mIU} / \mathrm{mL}$} \\
\hline
\end{tabular}

TP: total protein, Alb: albumin, T-Bil: total bilirubin, BUN: blood urea nitrogen, Cr: creatinine, AST: asparatate aminotransferase, ALT: alanine aminotransferase, LDH: lactate dehydrogenase, $\gamma$-GTP: $\gamma$-glutamyl transpeptidase, CRP: C-reactive protein, WBC: white blood cell, $\mathrm{Hb}$ : hemoglobin, RBC: red blood cell, $\mathrm{Ht}$ : hematocrit, Plt: platelet, EPO: erythropoietin Normal range of EPO: 9.1-32.8 mIU/mL myeloid hematologic disorders characterized by features of chronic inflammation (14), the relationship between PV and BD has not been clarified. There is a case report of sigmoid sinus thrombosis presenting with posterior alexia in a patient with BD and PV (15), but some reports have suggested that JAK2 mutations are not associated with thrombosis in BD $(16,17)$.

We herein report a case of intestinal Behçet's-like disease associated with PV and trisomy 8. To our knowledge, this is the first report of the co-existence of $\mathrm{PV}$ and intestinal Behçet's-like disease with trisomy 8.

\section{Case Report}

A 70-year-old woman presented to our hospital with increased white blood cell (WBC) counts and hematocrit levels. She had flushing, but her spleen was not palpable. Blood examinations showed a hemoglobin ( $\mathrm{Hb}$ ) level of $18.0 \mathrm{~g} / \mathrm{dL}$, platelet (Plt) count of $48.1 \times 10^{4} / \mu \mathrm{L}$, erythropoietin (EPO) $8.4 \mathrm{mIU} / \mathrm{mL}$ (normal range: 9.1-32.8 $\mathrm{mIU} / \mathrm{mL}$ ), and WBC count of $20,560 / \mu \mathrm{L}$, along with a serum lactate dehydrogenase (LDH) level of $397 \mathrm{U} / \mathrm{L}$ (Table 1) . A G-banded chromosome analysis revealed trisomy 8 and 9 (Fig. 1). A $J A K 2$ V617F mutation was identified in the peripheral leukocytes using a polymerase chain reaction (PCR) -direct sequencing analysis. Based on these findings, she was diagnosed with PV in 2012 in accordance with the World Health Organization classification. Abdominal ultrasonography revealed portal vein thrombosis during treatment with phlebotomy, aspirin, and hydroxyurea (HU). She remained clinically asymptomatic and showed no significant changes in clinical examination findings for the next five years.

Five years after the diagnosis of PV, she complained of bloody stool. Ileocolonoscopy was conducted and revealed

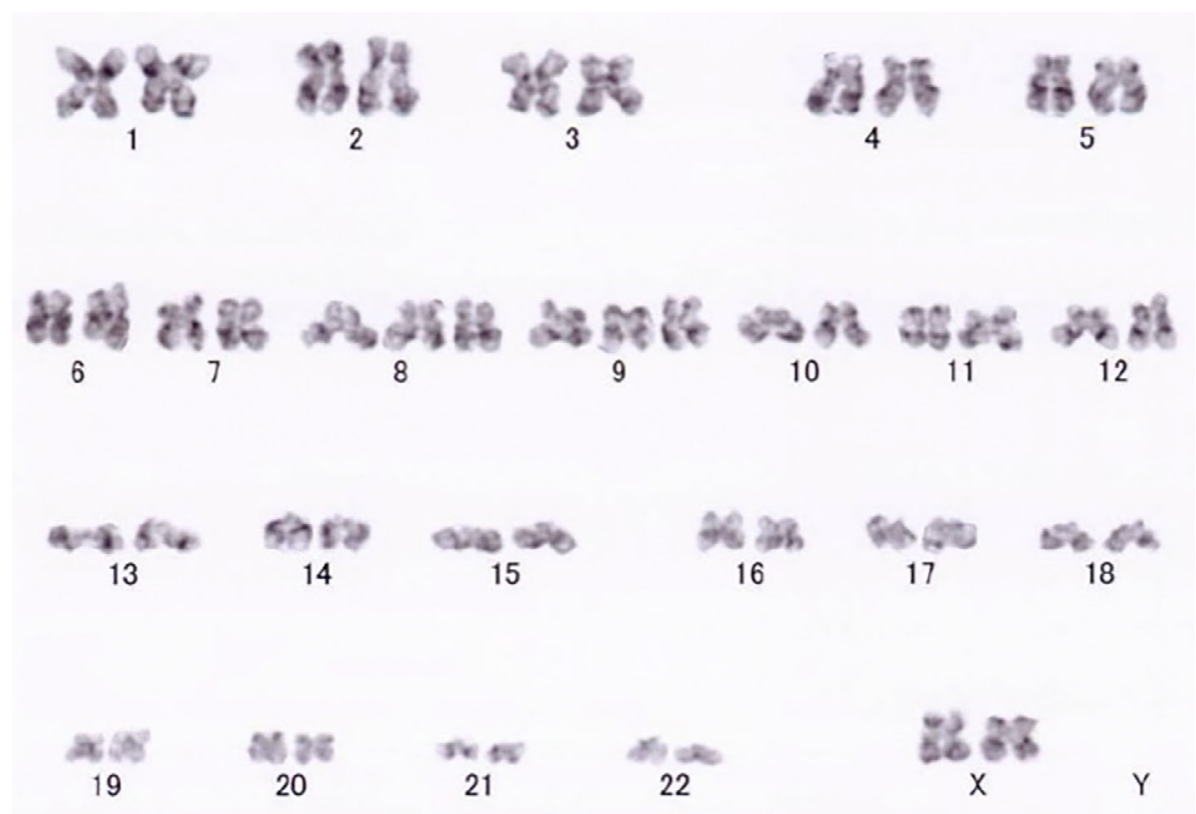

Figure 1. G-banded chromosome analysis showing the trisomy 8 and 9. 
A
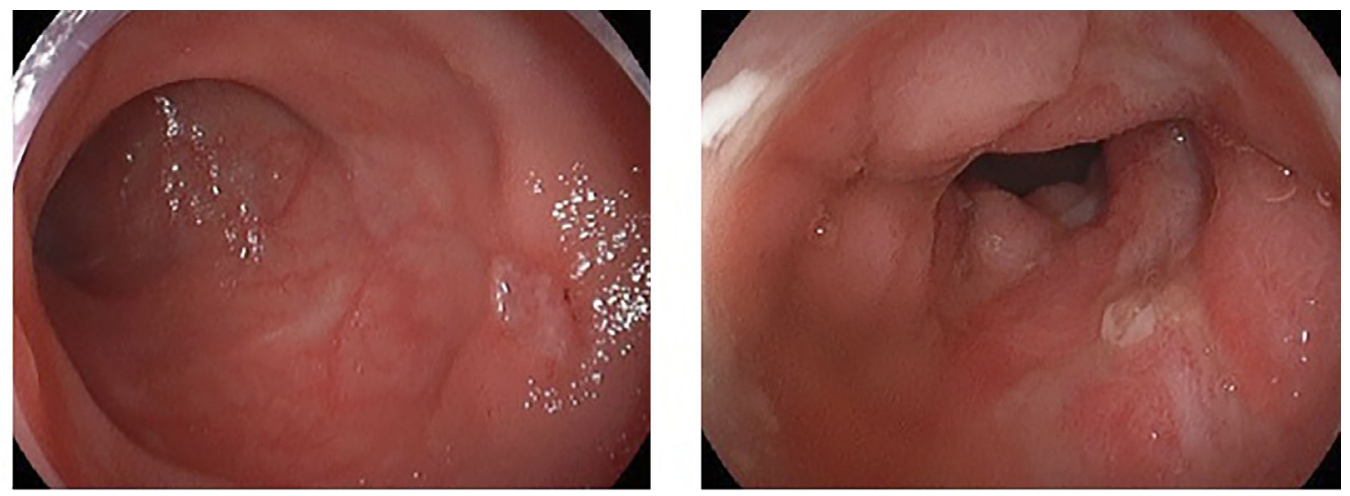

B

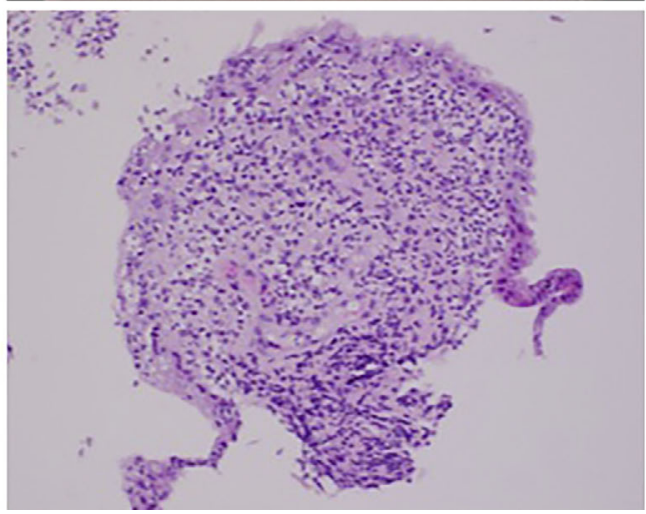

C

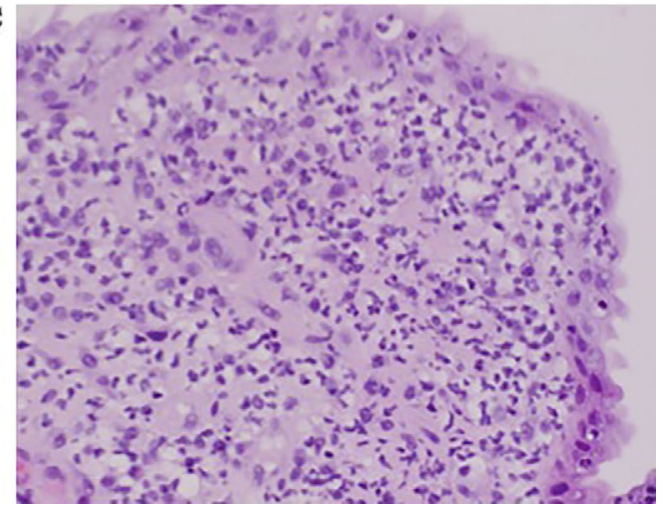

Figure 2. Endoscopic images showing multiple punched-out ulcers in the ileocecal region (A). Histological images of biopsy specimens from the margin of the ileocecal ulcers showed no signs of cancer. There were only non-specific inflammatory changes at (B) $20 \times$ magnification of the objective lens and (C) 40x magnification; the magnification of the ocular lens is not identified because camera systems have become an integral part of the microscope (Hematoxylin and Eosin staining).

Table 2. Patient Laboratory Data on Admission.

\begin{tabular}{|c|c|}
\hline Test name & Value \\
\hline \multicolumn{2}{|l|}{ Biochemistry } \\
\hline TP & $7.3 \mathrm{~g} / \mathrm{dL}$ \\
\hline $\mathrm{Alb}$ & $3.3 \mathrm{~g} / \mathrm{dL}$ \\
\hline T-Bil & $0.4 \mathrm{mg} / \mathrm{dL}$ \\
\hline BUN & $16.1 \mathrm{mg} / \mathrm{dL}$ \\
\hline $\mathrm{Cr}$ & $0.82 \mathrm{mg} / \mathrm{dL}$ \\
\hline AST & $17 \mathrm{IU} / \mathrm{L}$ \\
\hline ALT & $14 \mathrm{IU} / \mathrm{L}$ \\
\hline LDH & $199 \mathrm{IU} / \mathrm{L}$ \\
\hline$\gamma$-GTP & $38 \mathrm{mg} / \mathrm{dL}$ \\
\hline CRP & $2.68 \mathrm{mg} / \mathrm{dL}$ \\
\hline $\mathrm{Na}$ & $133 \mathrm{mEq} / \mathrm{L}$ \\
\hline K & $5.1 \mathrm{mEq} / \mathrm{L}$ \\
\hline $\mathrm{Cl}$ & $100 \mathrm{mEq} / \mathrm{L}$ \\
\hline \multicolumn{2}{|l|}{ Hematology } \\
\hline WBC & $13,270 / \mu \mathrm{L}$ \\
\hline $\mathrm{Hb}$ & $10.5 \mathrm{~g} / \mathrm{dL}$ \\
\hline $\mathrm{RBC}$ & $335 \times 10^{4} / \mu \mathrm{L}$ \\
\hline $\mathrm{Ht}$ & $34.1 \%$ \\
\hline Plt & $83.1 \times 10^{4} / \mu \mathrm{L}$ \\
\hline
\end{tabular}

multiple ulcers in the ileocecal region (Fig. 2A) . A histopathological examination revealed no signs of cancer or non-specific inflammation (Fig. 2B, C) . On a physical ex- amination, the patient was afebrile and had no ocular symptoms or genital ulcers. A blood analysis showed anemia with a red blood cell count of $3.35 \times 10^{4} / \mu \mathrm{L}, \mathrm{Hb}$ level of $10.5 \mathrm{~g} /$ $\mathrm{dL}$, hematocrit of $34.1 \%$, and Plt count of $83.1 \times 10^{4} / \mu \mathrm{L}$ (Table 2) . We initially suspected non-steroidal antiinflammatory drug (NSAID)-induced enteropathy and changed aspirin to cilostazol. Her anemia improved after the medication change, and we decided to monitor her condition.

Two years later, she presented with relapse of anemia, and ileocolonoscopy revealed recurrence of multiple ulcers in the ileocecal region (Fig. 3) . She presented again with bloody stool as well, and no improvement was found, so cilostazol and HU were withdrawn. Despite the cessation of cilostazol and HU, she again developed bloody stool. Given the lack of response to treatment and the typical endoscopic findings of segmental mucosal inflammation, punched-out, fissuring or aphthoid ulcers in the ileocecal area, we suspected complication with intestinal $\mathrm{BD}$, but the major criteria of $\mathrm{BD}$ were not present in this case.

Ileocolonoscopy revealed a few deep, oval-shaped ulcers in the ileocecal region (Fig. 4A) . A histopathological examination of tissue specimens suggested no signs of cancer or non-specific inflammation (Fig. 4B, C) . CMV infection was denied by tissue immunohistological staining and serum antigenemia. Polyarteritis nodosa and microscopic polyangiitis were denied by ANCA and Hematoxylin-Eosin staining. 

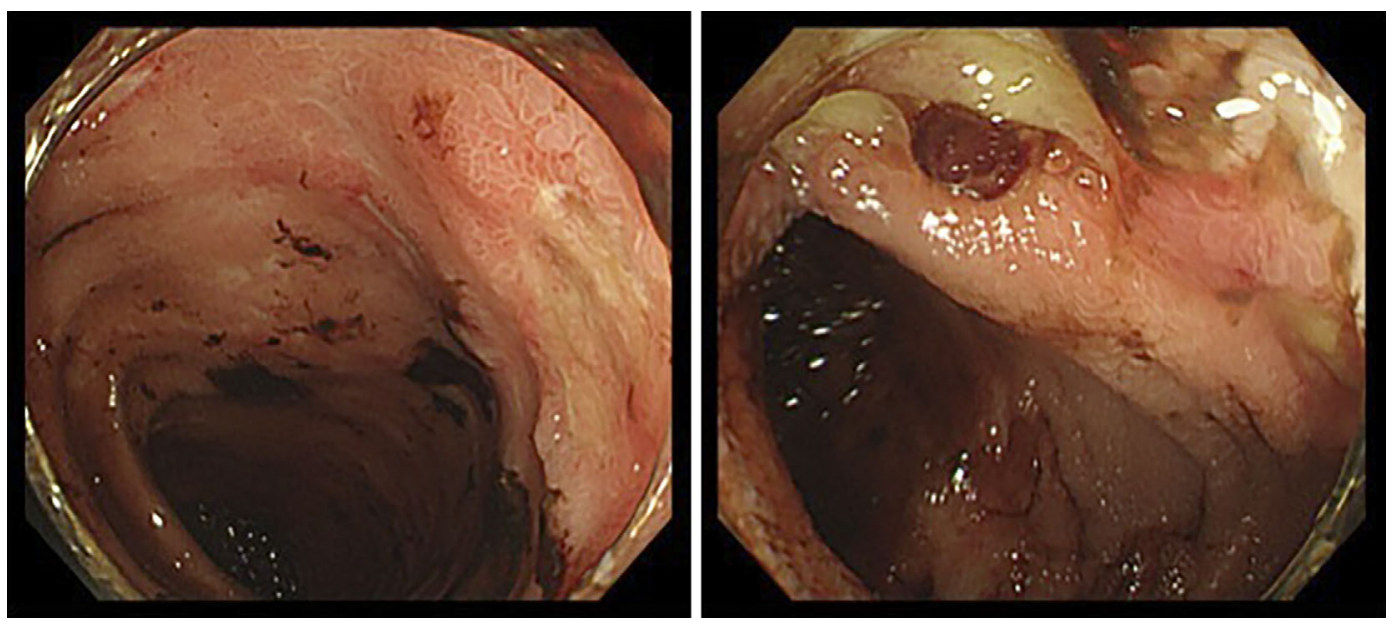

Figure 3. Endoscopic image showing multiple punched-out ulcers in the ileocecum (A). Endoscopic image showing a well-circumscribed ulcer in the ileocecum (B).
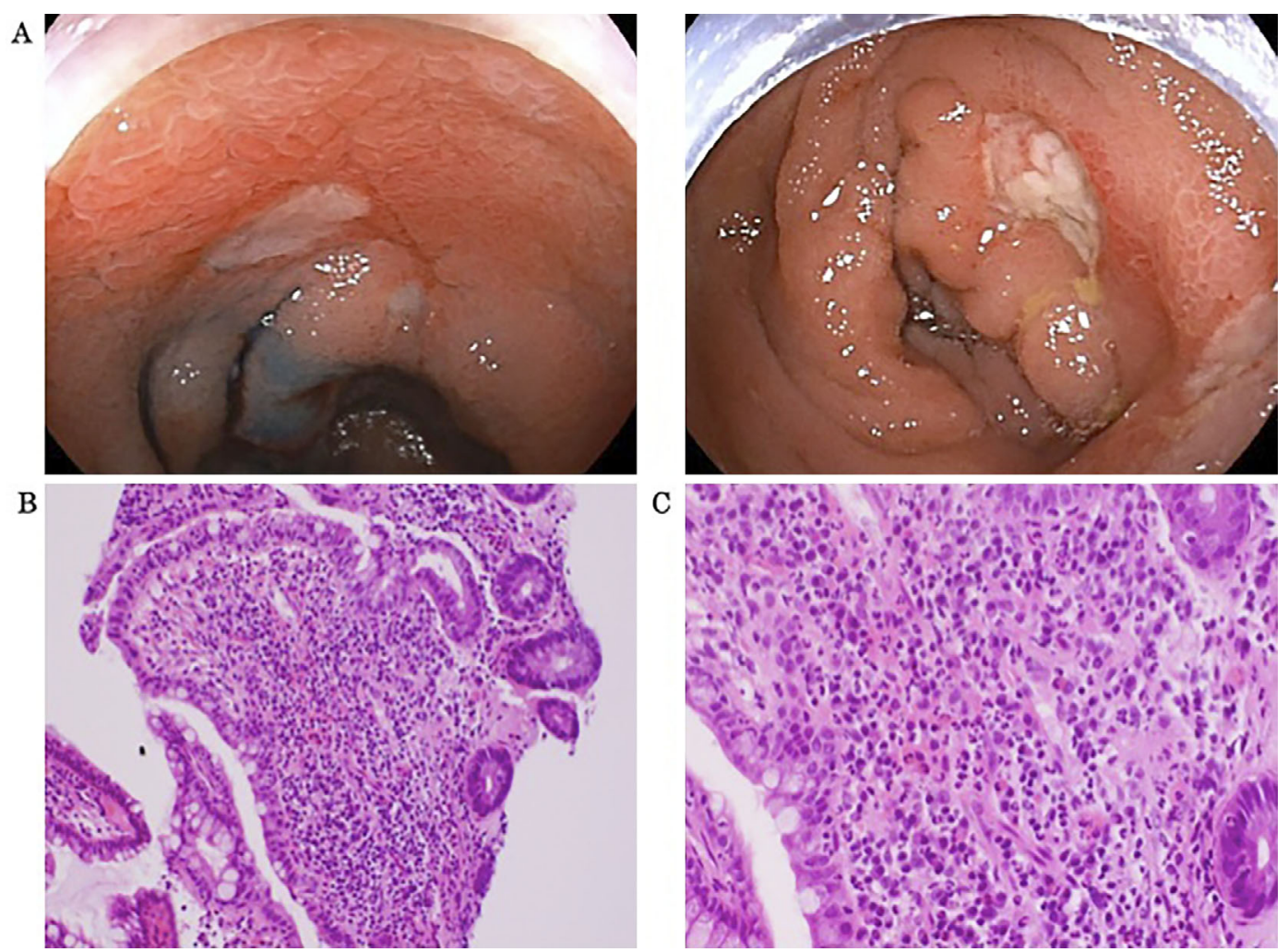

Figure 4. Endoscopic images showing multiple punched-out ulcers in the ileocecum (A). Histological images of biopsy specimens from the margin of the ileocecal ulcers showed no signs of cancer. There were only non-specific inflammatory changes at (B) 20x magnification of the objective lens and (C) 40× magnification (Hematoxylin and Eosin staining).

She was negative for the HLA-B51 antigen.

Recent studies have suggested that trisomy 8 may lead to the development of intestinal ulcers in patients with myeloid diseases, and BD is also a well-known chronic inflammatory disease associated with myeloid diseases. Based on these findings and colonoscopic findings resembling those of $\mathrm{BD}$, she was diagnosed with intestinal Behçet's-like disease complicated with PV with trisomy 8.

We prescribed oral prednisolone (PSL), which resulted in symptom improvement (Fig. 5). She underwent steroid therapy with $40 \mathrm{mg} / \mathrm{day}$ of PSL, and the dose was reduced to $5 \mathrm{mg} /$ day at 2 months later. After five months of therapy, ileocolonoscopy revealed improvement of bowel inflammation (Fig. 6). The treatment of has PSL contributed to the control of her intestinal Behçet's-like disease, and she remains recurrence-free since completing PSL therapy. 
on admission

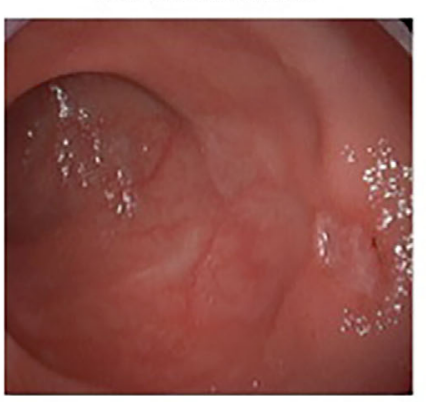

on readmission

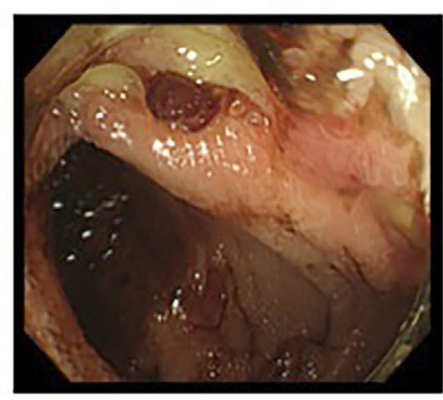

at recurrence

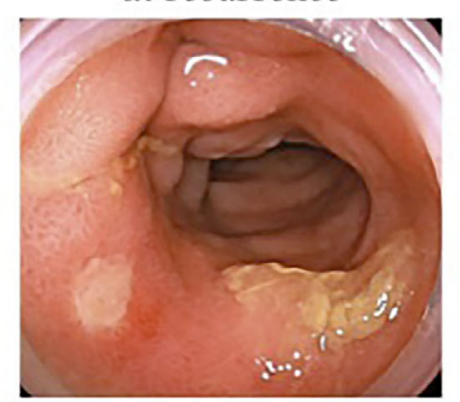

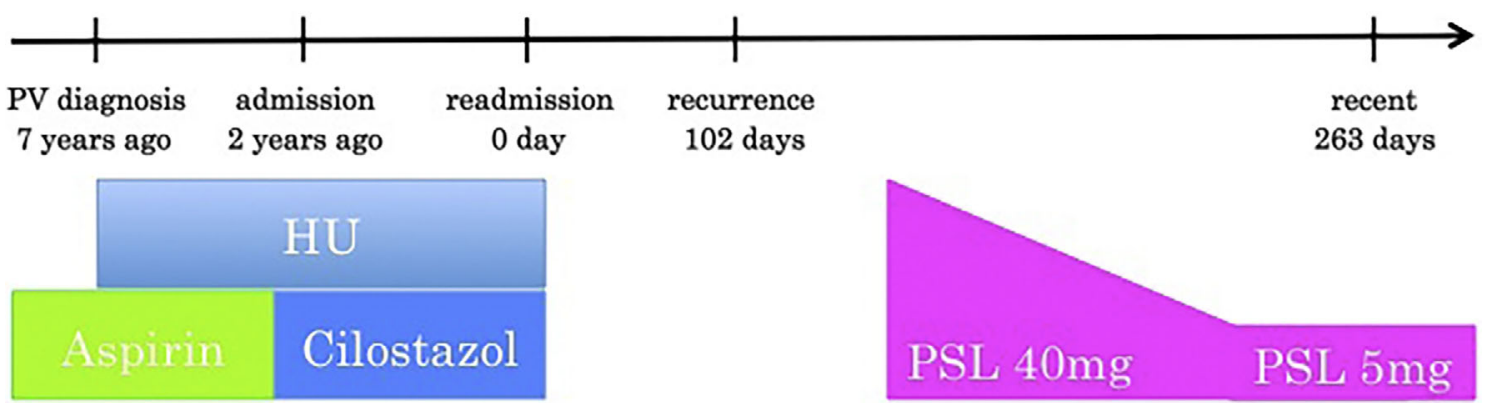

Figure 5. Therapy flow chart. First, she was diagnosed with PV and started treatment with phlebotomy, aspirin, and hydroxyurea. Five years after diagnosis, she had bloody stools and ileocolonoscopy revealed multiple ulcers in the ileocecal region. At first we suspected NSAIDs-induced enteropathy and monitored her condition while changing aspirin to cilostazol. No improvement was found, so cilostazol and HU were withdrawn. Since drug withdrawal was not effective, she was diagnosed with intestinal Behçet's-like disease associated PV with trisomy 8 and started administration PSL on recurrence. PSL was effective and tapered slowly over several weeks. " 0 day" refers to the day of readmission, "102 day" refers to the day of recurrence. "263 days" refers to the day of the most recent ileocolonoscopy after recurrence. PV: polycythemia vera, NSAIDs: non-steroidal anti-inflammatory drugs, HU: hydroxyurea, PSL: prednisolone
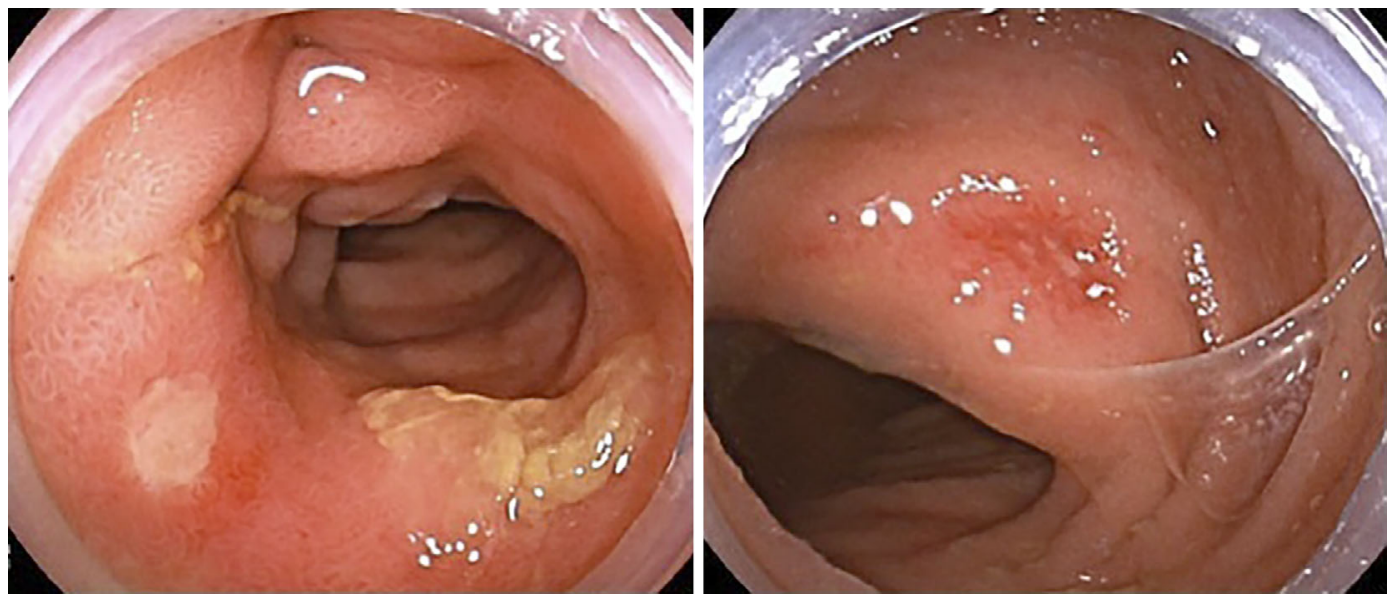

Figure 6. The most recent endoscopic images after recurrence. Ileocolonoscopy revealed improvement of intestinal Behçet's-like disease.

\section{Discussion}

The development of chronic inflammatory disease is a rare but significant complication of myeloid malignancies, especially in MDS. MDS is sometimes complicated with autoimmune diseases or Sweet syndrome. BD is also a wellknown chronic inflammatory disease associated with myeloid diseases (9-11). Interestingly, a previous case report highlighted the presence of a trisomy 8 chromosomal abnormality in a BD patient associated with myeloid diseases (18). In contrast, the association of BD and MPN, an- 
other form of myeloid disease, is quite rare. We herein report a patient with PV and trisomy 8 who developed intestinal Behçet's-like disease five years after the diagnosis of PV.

MPNs, including PV, essential thrombocythemia (ET), and PMF, are characterized by the presence of a driver mutation of JAK2. In PV, $90 \%$ of cases have the JAK2V617F mutation, and another $5 \%$ have a JAK2 mutation in exon 12 of the gene. It is well recognized that aberrant activation of JAK2 in MPN cells causes a chronic inflammatory state through the disruption of immune signaling networks, which leads to the overproduction of inflammatory cytokines, including IL-1 $\beta$, IL-8, and tumor necrosis factor-alpha (TNF$\alpha$ ), by both neoplastic clones and bystander immune cells (14). Indeed, MPN patients are more likely to develop autoimmune diseases than others (19). Although speculative at present, these impaired immune responses might be responsible for the development of BD in MPN.

Modulation of the microbiome in MPN patients might also play an important role in the development of BD associated with MPN, since the role of dysbiosis in autoimmune diseases, such as inflammatory bowel disease (IBD), including Crohn's disease and ulcerative colitis (UC), is now well recognized $(20,21)$. Indeed, Fleischman et al. revealed that the amount of Prevotellaceae family is increased in MPN patients compared with normal control patients, and the cytokines TNF- $\alpha$ and IL-17a explained most of the variance in microbiome data (14).

The presence of trisomy 8 also facilitates the production of inflammatory cytokines, such as TNF- $\alpha$, IL-6, and IL-1 $\beta$, which promote the neutrophil function and are known risk factors for intestinal ulcers (9). Tada et al. reported that trisomy 8 might lead to the development of intestinal ulcers in patients with MDS (22). Controlling MDS-related BD is difficult, as the underlying immune abnormalities arise from MDS itself (23). The precise role of trisomy 8 is unknown, but it is suggested that genes located on chromosome 8 predispose MDS patients to develop BD. Although an association between intestinal BD and MPN has not yet been reported, a similar mechanism is predicted in trisomy 8 cases. Trisomy 8 may thus lead to the development of intestinal Behçet's-like disease in patients with MPN. The genetic background also affects the pathogenesis underlying the relationship between MPN and IBD, given that the JAK2 46/1 haplotype identified as associated with JAK2-mutated MPN is also associated with Crohn's disease (24).

It should be noted that intestinal Behçet's-like disease associated with myeloid malignancy has unique clinical findings. The frequency of intestinal Behçet's-like disease is significantly higher in $\mathrm{BD}$ patients with bone marrow failure than in patients without bone marrow failure $(61.5 \%$ vs. $13.6 \%$ ) (25). Handa et al. reported the data of 64 patients with BD and MDS, among which $80 \%$ had trisomy 8 , and observed a high frequency of intestinal lesions (66\%), whereas the incidence of ocular lesions was low (13\%). These observations suggested that intestinal lesions are a characteristic finding in $\mathrm{BD}$ associated with trisomy 8 and bone marrow failure, especially in MDS (26).

Although treatment for BD typically involves immunosuppressive agents, such as cyclosporine, cyclophosphamide, anti-TNF- $\alpha$ antibody (infliximab, adalimumab), and corticosteroids, a standard treatment for BD associated with myeloid neoplasms has not yet been established. Previous reports indicated that the intestinal lesions in BD patients with trisomy 8 are refractory to conventional therapy (11). The administration of immunosuppressive agents alone is insufficient to control MDS-associated intestinal BD, and many patients die of hemorrhaging (27), infection (28), or recurrent ulcers (29) before MDS treatment. Similar to MDS-related BD $(23,30)$, controlling MPN-associated BD is assumed to be difficult, as the underlying immune abnormalities arise from MPN itself. Surgery is considered for patients with intestinal BD who are unresponsive to medical treatment or those with bowel complications, such as perforation or persistent bleeding (31). Very recently, tofacitinib, a non-selective JAK inhibitor, was approved for moderateto-severe ulcerative colitis refractory or intolerant to TNF inhibitors (32). Interestingly, ruxolitinib, an inhibitor of JAK1/ JAK2, is widely used to treat PV and PMF and may be attractive for use in treating MPN patients with BD (33). However, Narazaki et al. reported a patient with trisomy 8positive PMF with $\mathrm{BD}$ who developed intestinal perforation during treatment with ruxolitinib (33). Therefore, caution should be practiced when using ruxolitinib in MPN patients with intestinal BD.

HLA-B51 confers the strongest genetic risk to BD (34). A genome-wide association study and meta-analysis identified common variants in interleukin 10 (IL-10) and at the IL-23 R-IL-12RB2 locus that predispose individuals to BD. Expression studies have shown that the disease-associated IL10 variants are associated with a reduced expression of this anti-inflammatory cytokine, which may lead to a susceptible inflammatory state, thus increasing susceptibility to BD (35). Our patient had HLA-B51-negative intestinal Behçet's-like disease, so a mutation in JAK2 or the presence of trisomy 8 may have facilitated the production of inflammatory cytokines and led to the development of intestinal Behçet's-like disease. A recent study reported that tofacitinib would be the first JAK inhibitor made available for treatment of ulcerative colitis (36). Tofacitinib acts on a multitude of cytokines at the same time by inhibiting the JAK/ STAT pathway, resulting in a wider effect on gastrointestinal inflammation then with other agents.

In conclusion, we reported a case of intestinal Behçet'slike disease complicated with PV and trisomy 8. The diagnosis and treatment of such patients may be complicated, but we believe that an improved understanding of this complex disease and collaborative therapy affecting key cellular regulators (including JAK2 inhibitors as well as other drugs) can lead to improved care and a reduced rate of adverse outcomes.

The authors state that they have no Conflict of Interest (COI). 


\section{Acknowledgement}

We would like to thank Enago (www.enago.jp) for the English language review.

\section{Financial Support}

This research received no specific grant from any funding agency in the public, commercial, or not-for-profit sectors.

\section{Human Rights}

All procedures followed have been performed in accordance with the ethical standards laid down in the 1964 Declaration of Helsinki and its later amendments.

\section{References}

1. James DG. Behcet's syndrome. N Engl J Med 301: 431-432, 1979.

2. Kaklamani VG, Vaiopoulos G, Kaklamanis PG. Behçet's disease. Semin Arthritis Rheum 27: 197-217, 1998.

3. Sakane T, Takeno M, Suzuki N, Inaba G. Behçet's disease. N Engl J Med 341: 1284-1291, 1999.

4. Dilşn N, Koniçe M, Aral O, Ocal L, Inanç M, Gül A. Comparative study of the skin pathergy test with blunt and sharp needLes in Behçet's disease: confirmed specificity but decreased sensitivity with sharp needLes. Ann Rheum Dis 52: 823-825, 1993.

5. Barešić M, Reihl M, Habek M, Vukojević N, Anić B. Improvement of neurological and ocular symptoms of Behçet's disease after the introduction of infliximab. Rheumatol Int 38: 1301-1306, 2018.

6. Adachi Y, Tsutsumi A, Murata H, et al. Behçet's disease accompanied by myelodysplastic syndrome with trisomy 8: two case reports and review of 15 Japanese cases. Mod Rheumatol 13: 90-94, 2003.

7. KaloterakisA, StavrianeasNG, KaragianniIN, etal. AdamantiadesBehçet's disease coexisting with acute myeloblastic leukaemia. Br J Dermatol 137: 317-318, 1997.

8. Narazaki T, Shiratsuchi M, Tsuda M, et al. Intestinal Behçet's disease with primary myelofibrosis involving trisomy 8. Acta Haematol 142: 253-256, 2019.

9. Tada Y, Koarada S, Haruta Y, et al. The association of Behçet's disease with myelodysplastic syndrome in Japan: a review of the literature. Clin Exp Rheumatol 24 (5 Suppl 42): S115-S119, 2006.

10. Kawabata H, Sawaki T, Kawanami T, et al. Myelodysplastic syndrome complicated with inflammatory intestinal ulcers: significance of trisomy 8. Intern Med 45: 1309-1314, 2006.

11. Toyonaga $T$, Nakase $H$, Matsuura $M$, et al. Refractoriness of intestinal Behçet's disease with myelodysplastic syndrome involving trisomy 8 to medical therapies - our case experience and review of the literature. Digestion 88: 217-221, 2013.

12. Chen G, Zeng W, Miyazato A, et al. Distinctive gene expression profiles of CD34 cells from patients with myelodysplastic syndrome characterized by specific chromosomal abnormalities. Blood 104: 4210-4218, 2004.

13. Spivak Jerry L. How I treat polycythemia vera. Blood 134: 341 352, 2019.

14. MendezLuque LF, Blackmon AL, Ramanathan G, Fleischman AG. Key role of inflammation in myeloproliferative neoplasms: instigator of disease initiation, progression and symptoms. Curr Hematol Malig Rep 14: 145-153, 2019.

15. Thomas P, Keightley A, Kamble R, Gunasekera N, Johnson MR. Sigmoid sinus thrombosis presenting with posterior alexia in a patient with Behçet's disease and polycythaemia: a case report.. J Med Case Rep 2: 175, 2008.

16. Adeeb F, Tayel M, El Kaffash DM, Idris KM, Hassan MF, Fraser AD. Janus kinase $2 \mathrm{~V} 617 \mathrm{~F}$ mutation and thrombotic events in
Behcet's disease: The Alexandria experience. Eur J Rheumatol 3: 73-74, 2016

17. Ar MC, Hatemi G, Ekizoğlu S, et al. JAK2V617F Mutation is not associated with thrombosis in behcet syndrome. Clin Appl Thromb Hemost 18: 421-426, 2012.

18. Shudan Wang, Noam Broder, Paula Marchetta, Johannes Nowatzky. Myelodysplastic syndrome presenting as a Behçet's-like disease with aortitis. BMJ Case Rep published online First [please include Day Month Year].

19. Kristinsson SY, Landgren O, Samuelsson J, Bjorkholm M, Goldin LR. Autoimmunity and the risk of myeloproliferative neoplasms. Haematologica 95: 1216-1220, 2010.

20. Sokol H, Pigneur B, Watterlot L, et al. Faecalibacterium prausnitzii is an anti-inflammatory commensal bacterium identified by gut microbiota analysis of Crohn disease patients. Proc Natl Acad Sci USA 105: 16731-16736, 2008.

21. Sokol H, Leducq V, Aschard $\mathrm{H}$, et al. Fungal microbiota dysbiosis in IBD. Gut 66: 1039-1048, 2017.

22. Tada Y, Koarada S, Haruta Y, Mitamura M, Ohta A, Nagasawa K. The association of Behcet's disease with myelodysplastic syndrome in Japan: a review of the literature. Clin Exp Rheumatol 24 (5 Suppl 42): S115-S119, 2006.

23. Ogawa $H$, Kuroda $T$, Inada $M$, et al. Intestinal Behcet's disease associated with myelodysplastic syndrome with chromosomal trisomy $8-$ a report of two cases and a review of the literature. Hepatogastroenterology 48: 416-420, 2001.

24. Barrett JC, Hansoul S, Nicolae DL, et al. Genome-wide association defines more than 30 distinct susceptibility loci for Crohn's disease.. Nat Genet 40: 955-962, 2008.

25. Ahn JK, Cha HS, Koh EM, et al. Behcet's disease associated with bone marrow failure in Korean patients: clinical characteristics and the association of intestinal ulceration and trisomy 8. Rheumatology 47: 1228-1230, 2008.

26. Handa T, Nakatsue T, Baba M, Takada T, Nakata K, Ishii H. Clinical features of three cases with pulmonary alveolar proteinosis secondary to myelodysplastic syndrome developed during the course of Behçet's disease. Respir Investig 52: 75-79, 2014.

27. Handa T, Arai Y, Mitani K. Myelodysplastic syndrome associated with intestinal tract-type Behçet disease characterized by an esophageal ulcer. J Clin Hematol 45: 135-137, 2004.

28. Tsubata R, Suzuki F, Sugihara T, et al. An autopsy case of intestinal Behçet's disease with sacroiliitis accompanied by myelodysplastic syndrome with trisomy 8. Jpn J Clin Immunol 28: 48-55, 2005.

29. Kimura S, Kuroda J, Akaogi T, Hayashi H, Kobayashi Y, Kondo M. Trisomy 8 involved in myelodysplastic syndromes as a risk factor for intestinal ulcers and thrombosis-Behçet's syndrome. Leuk Lymphoma 42: 115-121, 2001.

30. Niwa Y, Miyake S, Sakane T, Shingu M, Yokoyama M. Autooxidative damage in Behçet's disease-endothelial cell damage following the elevated oxygen radicals generated by stimulated neutrophils. Clin Exp Immunol 49: 247-255, 1982.

31. Yuki M, Fumihiko I, Yasuaki I, et al. Long-term outcome after surgery in a patient with intestinal Behçet's disease complicated by myelodysplastic syndrome and trisomy 8 . Intest Res [published online ahead of print, 2020 May 12].

32. Agrawal M, Kim ES, Colombel JF. JAK inhibitors safety in ulcerative colitis: practical implications. J Crohns Colitis 14 (Supplement_2): S755-S760, 2020.

33. Narazaki T, Shiratsuchi M, Tsuda M, et al. Intestinal Behçet's disease with primary myelofibrosis involving trisomy 8 . Acta Haematol 142: 253-256, 2019.

34. Ohno S, Ohguchi M, Hirose S, et al. Close association of HLABw51 with Behçet's disease. Archives of Ophthalmology 100: 1455-1458, 1982.

35. Remmers EF, Cosan F, Kirino Y, et al. Genome-wide association 
study identifies variants in the MHC class I, IL10, and IL23R-IL 12RB2 regions associated with Behçets disease. Nat Genet 42: 698, 2010.

36. Agnès Fernández-Clotet, Jesús Castro-Poceiro, Julián Panés. Tofacitinib for the treatment of ulcerative colitis. Expert Review of Clinical Immunology 14: 11, 881-892, 2018.
The Internal Medicine is an Open Access journal distributed under the Creative Commons Attribution-NonCommercial-NoDerivatives 4.0 International License. To view the details of this license, please visit (https://creativecommons.org/licenses/ by-nc-nd/4.0/).

(C) The Japanese Society of Internal Medicine Intern Med Advance Publication 